\title{
On the Solution of One-Dimensional Ising Models
}

\author{
Yu. N. Kharchenko \\ Institute for Applied Mathematics, Far Eastern Branch of Russian Academy Sciences, Vladivostok, Russia \\ Email: har@iam.dvo.ru
}

How to cite this paper: Kharchenko, Yu.N. (2018) On the Solution of One-Dimensional Ising Models. Journal of Applied Mathematics and Physics, 6, 960-967. https://doi.org/10.4236/jamp.2018.65082

Received: April 8, 2018

Accepted: May 13, 2018

Published: May 16, 2018

Copyright (C) 2018 by author and Scientific Research Publishing Inc. This work is licensed under the Creative Commons Attribution International License (CC BY 4.0).

http://creativecommons.org/licenses/by/4.0/

\begin{abstract}
In this paper it is shown that the thermodynamic limit of the partition function of the statistical models under consideration on a one-dimensional lattice with an arbitrary finite number of interacting neighbors is expressed in terms of the principal eigenvalue of a matrix of finite size. The high sparseness of these matrices for any number of interactions makes it possible to perform an effective numerical analysis of the macro characteristics of these models.
\end{abstract}

\section{Keywords}

Ising Model, Transfer Matrix, Statistical Sum, Free Energy, Singular Curves, Phase Transitions

\section{Introduction}

The data of modern studies of the magnetic properties of monoatomic chains [1] [2] raise the question of choosing a model for describing these phenomena and how to solve it. Here we study the problems of solving translationally invariant models with a binary interaction of spins located at the nodes of a one-dimensional lattice.

\section{The Partition Function}

Let $\sigma_{n}^{m}, m=1, \cdots, M, n=1, \cdots, N+M$ be the binary variables (spins) that take two values of \pm 1 , and $M, N, M<N$ are some natural numbers. We consider a statistical model on a one-dimensional lattice, with nodes numbered by natural numbers $1, \cdots, N+M$, whose partition function has the form

$$
Z_{N}^{(1)}(M)=Z_{N}^{(1)}\left(M,\left\{K_{i}\right\}, H\right)=\sum_{\sigma^{1}} \prod_{j=1}^{N} \exp \left(\sum_{i=1}^{M} K_{i} \sigma_{j}^{1} \sigma_{j+i}^{1}+H \sigma_{j}^{1}\right) .
$$


Here summation over $\sigma^{1}=\left(\sigma_{1}^{1}, \sigma_{2}^{1}, \cdots, \sigma_{N}^{1}\right)$ means summation over all states of the spins, $K_{i}, i=1, \cdots, M$ are some real parameters of the interspin interaction, $H$ is the parameter of interaction with the external field, and the boundary condition $\sigma_{N+j}^{1}=\sigma_{j}^{1}, j=1, \cdots, M$ is satisfied. We are interested in the free energy $F^{(1)}(M)$, which for a specific value of $M$ is calculated by the formula

$$
F^{(1)}(M)=F^{(1)}\left(M,\left\{K_{i}\right\}, H\right)=\lim _{N \rightarrow \infty} \frac{\ln Z_{N}^{(1)}\left(M,\left\{K_{i}\right\}, H\right)}{N} .
$$

For $M=1$, the Formula (1) gives the partition function of the one-dimensional Ising model with the interaction between nearest spins, which has an exact solution. The free energy of this model is calculated through the principal eigenvalue (PEV) (positive single and maximum modulo eigenvalues) of a matrix of size $2 \times 2$ [3] [4] (Ising solution). We show that the free energy of a model with an arbitrary value of $M$ is calculated through the PEV of a matrix of size $2^{M} \times 2^{M}$ (this eigenvalue exists for finite values of $M$ by the Perron-Frobenius theorem). We call such models one-dimensional Ising models.

Let $\mu_{n}=\left(\sigma_{n}^{1}, \sigma_{n}^{2}, \cdots, \sigma_{n}^{M}\right)$ be a set of binary variables. We define the value of $g\left(\mu_{j}, \mu_{j+1}\right)$ by the formula

$$
g\left(\mu_{j}, \mu_{j+1}\right)=\exp \left(\sum_{i=1}^{M} K_{i} \sigma_{j}^{1} \sigma_{j+1}^{i}+H \sigma_{j}^{1}\right) \delta\left(\sigma_{j}^{2}, \sigma_{j+1}^{1}\right) \cdots \delta\left(\sigma_{j}^{M}, \sigma_{j+1}^{M-1}\right),
$$

where $\delta\left(\sigma_{j}^{i}, \sigma_{j+1}^{i-1}\right)$ are the Kronecker symbols, $\delta(x, y)=0, x \neq y$, $\operatorname{delta}(x, y)=1, x=y$, and $x, y$ are integers. Consider the sum $U^{(1)}(M)$, which has the form

$$
U^{(1)}(M)=\sum_{\sigma^{1}} \sum_{\sigma^{2}} \cdots \sum_{\sigma^{M}} \prod_{j=1}^{N} g\left(\mu_{j}, \mu_{j+1}\right),
$$

where the summation is over the sets of binary variables $\sigma^{i}=\left(\sigma_{1}^{i}, \sigma_{2}^{i}, \cdots, \sigma_{N}^{i}\right)$ and the boundary condition $\sigma_{N+1}^{i}=\sigma_{1}^{i}, i=1, \cdots, M$. Performing the summation over the sets $\left(\sigma^{2}, \sigma^{3}, \cdots, \sigma^{M}\right)$, we get the partition function (1), on the other hand, the sum $U^{(1)}(M)$ can be written in the form

$$
U^{(1)}(M)=\sum_{\mu_{1}} \sum_{\mu_{2}} \cdots \sum_{\mu_{N}} \prod_{j=1}^{N} g\left(\mu_{j}, \mu_{j+1}\right) .
$$

In this case, we can represent the set of values of each value $g\left(\mu_{j}, \mu_{j+1}\right)$ as the set of values of elements of some matrix $A(M)$ of size $2^{M} \times 2^{M}$ enumerated by the values of the sets $\mu_{j}, \mu_{j+1}$. Hence we obtain

$$
U^{(1)}(M)=Z_{N}^{(1)}(M)=\operatorname{tr}\left(A(M)^{N}\right) .
$$

The matrix $A(M)$ is called the transfer matrix, the free energy $F^{(1)}(M)$ (2) is expressed in terms of the PE $\lambda(A(M))$ transfer-matrix $A(M)$ by the formula [3] [4].

$$
F^{(1)}(M)=\ln (\lambda(A(M)))
$$

Next, the transfer matrix $A(M)$ will be expressed in the form convenient for applications. To do this, we present some information from the theory of in- 
dexed matrices.

\section{Indexed Matrices}

The theory of indexed matrices for lattice models is presented in the monograph [2]. We define single-index matrices, permutation operators for indices, and formulas that will be needed later.

The operator of permutations of indices is the operator permuting the canonical basis of the Euclidean space $R^{2^{J}} \quad \delta^{j}=\left[\delta(j, 1), \cdots, \delta\left(j, 2^{J}\right)\right]^{\mathrm{T}}$ (Here $J$ is a positive integer and $\mathrm{T}$ is a transpose sign) in the following order: $\delta^{j} \rightarrow \delta^{2 j-1}$ and $\delta^{2^{J-1}+j} \rightarrow \delta^{2 j}, j=1, \cdots, 2^{J-1}$. Its matrix $2^{J} \times 2^{J}$ in this basis will be denoted by the symbol $P_{J}$.

Let $q$ be a number matrix of size $2 \times 2$, then we denote by $q_{[1]}$ a block-diagonal matrix of size $2^{N} \times 2^{N}$ whose blocks are the matrix $q$. The indexed matrix $q_{[j]}$ is defined by the formula $q_{[j]}=P_{J}^{j} q_{[1]} P_{J}^{-j}$. The matrix $q_{[j]}$ is a block-diagonal matrix whose blocks are the matrix obtained by replacing the identity matrices $2^{(j-1)} \times 2^{(j-1)}$ with each element of the matrix $q$, multiplied by the value of this element.

For concrete matrices of size $2 \times 2$, we use the notation $\{(*, *),(*, *)\}$, where we write row elements in parentheses.

It follows from the block structure of indexed matrices that the matrices $q$ and $\mathrm{w}$ commute with different indices

$$
q_{[i]} w_{[j]}=w_{[j]} q_{[i]}, i \neq j,
$$

and from the definition of indexed matrices it follows that

$$
q_{[i]} w_{[i]}=(q w)_{[i]} .
$$

We introduce the following notation for basis matrices of size $2 \times 2$

$$
\begin{aligned}
& e=e(+1,+1)=\{(1,0),(0,0)\}, f=e(+1,-1)=\{(0,1),(0,0)\}, \\
& g=e(-1,+1)=\{(0,0),(1,0)\}, h=e(-1,-1)=\{(0,0),(0,1)\} .
\end{aligned}
$$

Then any matrix $B$ of size $2^{J} \times 2^{J}$ can be written in the form

$$
B=\sum_{\sigma^{1}, \sigma^{2}} \beta\left(\sigma^{1}, \sigma^{2}\right) \prod_{j=1}^{J} e\left(\sigma_{j}^{1}, \sigma_{j}^{2}\right)_{[j]} \equiv B_{[J, \cdots, 1]},
$$

where $\beta\left(\sigma^{1}, \sigma^{2}\right)$-number of coefficients and the following equalities hold

$$
e\left(\sigma_{j}^{1}, \sigma_{j}^{2}\right)_{[j]} e\left(\sigma_{j}^{3}, \sigma_{j}^{4}\right)_{[j]}=\delta\left(\sigma_{j}^{2}, \sigma_{j}^{3}\right) e\left(\sigma_{j}^{1}, \sigma_{j}^{4}\right)_{[j]}, j=1, \cdots, J .
$$

The trace of the matrix $B$ is calculated as follows

$$
\begin{aligned}
\operatorname{tr}(B) & =\sum_{\sigma^{1}, \sigma^{2}} \beta\left(\sigma^{1}, \sigma^{2}\right) \operatorname{tr}\left(\prod_{j=1}^{J} e\left(\sigma_{j}^{1}, \sigma_{j}^{2}\right)_{[j]}\right) \\
& =\sum_{\sigma^{1}, \sigma^{2}} \beta\left(\sigma^{1}, \sigma^{2}\right) \prod_{j=1}^{J} \delta\left(\sigma_{j}^{1}, \sigma_{j}^{2}\right)=\sum_{\sigma^{1}} \beta\left(\sigma^{1}, \sigma^{1}\right) .
\end{aligned}
$$

In addition to the operator $P_{J}$, we also introduce permutation operators whose matrices $P_{[i, j]}$ have the form 


$$
P_{[i, j]}=e_{[i]} e_{[j]}+f_{[i]} g_{[j]}+g_{[i]} f_{[j]}+h_{[i]} h_{[j]}, i \neq j .
$$

They have the following properties

$$
q_{[i]} P_{[i, j]}=P_{[i, j]} q_{[j]}, P_{[i, j]} P_{[i, m]}=P_{[j, m]} P_{[i, j]}, P_{J}=P_{[J, 1]} P_{[J-1,1]} \cdots P_{[2,1]},
$$

in particular, the equalities

$$
P_{[J, j]} P_{[J, m]}=P_{[j, m]} P_{[J, j]}, q_{[j]} P_{[J, j]}=P_{[J, j]} q_{[J]}
$$

are satisfied.

We note that it follows from (8) that the matrix $B$ can be written in the form

$$
B=e_{[J]} C_{[J-1, \cdots, 1]}+f_{[J]} D_{[J-1, \cdots, 1]}+g_{[J]} E_{[J-1, \cdots, 1]}+h_{[J]} F_{[J-1, \cdots, 1]},
$$

where all the indexed matrices have the size $2^{J} \times 2^{J}$. But, proceeding from the block structure of indexed matrices, matrix $B$ can be given the form

$$
B=\left\{\left(C_{[J-1, \cdots, 1]}, D_{[J-1, \cdots, 1]}\right),\left(E_{[J-1, \cdots, 1]}, F_{[J-1, \cdots, 1]}\right)\right\},
$$

where the elements of a matrix of size $2 \times 2$ should be understood as matrices of size $2^{(J-1)} \times 2^{(J-1)}$. Thus, the equations (12), useful for our further calculations, in this notation take the form

$$
\begin{gathered}
\left\{\left(e_{[j]}, g_{[j]}\right),\left(f_{[j]}, h_{[j]}\right)\right\}\left\{\left(e_{[m]}, g_{[m]}\right),\left(f_{[m]}, h_{[m]}\right)\right\}=P_{[j, m]}\left\{\left(e_{[j]}, g_{[j]}\right),\left(f_{[j]}, h_{[j]}\right)\right\}, \\
q_{[j]}\left\{\left(e_{[j]}, g_{[j]}\right),\left(f_{[j]}, h_{[j]}\right)\right\}=\left\{\left(e_{[j]}, g_{[j]}\right),\left(f_{[j]}, h_{[j]}\right)\right\} q,
\end{gathered}
$$

where all the indexed matrices have the size $2^{(J-1)} \times 2^{(J-1)}$.

\section{Transfer Matrix}

For a given value of $M$, the specific form of the transfer matrix $A(M)$ is determined by the correspondence between the sets of binary variables and the natural numbers that number the elements of the matrix. Consider matrix, which is defined by formula

$$
A(M)=\sum_{\mu_{1}} \sum_{\mu_{2}} g\left(\mu_{1}, \mu_{2}\right) e\left(\sigma_{1}^{1}, \sigma_{2}^{1}\right)_{[1]} e\left(\sigma_{1}^{2}, \sigma_{2}^{2}\right)_{[2]} \cdots e\left(\sigma_{1}^{M}, \sigma_{2}^{M}\right)_{[M]},
$$

where $g\left(\mu_{1}, \mu_{2}\right)$ according to (3) has the form

$$
g\left(\mu_{1}, \mu_{2}\right)=\exp \left(\sum_{i=1}^{M} K_{i} \sigma_{1}^{1} \sigma_{2}^{i}+H \sigma_{1}^{1}\right) \delta\left(\sigma_{1}^{2}, \sigma_{2}^{1}\right) \cdots \delta\left(\sigma_{1}^{M}, \sigma_{2}^{M-1}\right),
$$

and all indexed matrices have the size $2^{M} \times 2^{M}$. Let us show that this matrix is the transfer matrix of the model (1). To do this, we calculate the partition function by the Formula (4). In these calculations, we use sets of binary variables $v_{n}=\left(v_{n}^{1}, v_{n}^{2}, \cdots, v_{n}^{M}\right)$ similar to $\mu_{n}$. First, we compute $A^{2}(M)$, for this we write $A^{2}(M)$ in the form

$$
\begin{aligned}
A^{2}(M)= & \sum_{\mu_{1}, \mu_{2}, \mu_{3}, v_{1}} g\left(\mu_{1}, \mu_{2}\right) e\left(\sigma_{1}^{1}, \sigma_{2}^{1}\right)_{[1]} \cdots e\left(\sigma_{1}^{M}, \sigma_{2}^{M}\right)_{[M]} g\left(v_{1}, \mu_{3}\right) \\
& e\left(v_{1}^{1}, \sigma_{3}^{1}\right)_{[1]} \cdots e\left(v_{1}^{M}, \sigma_{3}^{M}\right)_{[M]} .
\end{aligned}
$$

Taking into account the commutation relations (6) and the equalities (9), we 
write this product in the form

$$
\begin{aligned}
& A^{2}(M) \\
& =\sum_{\mu_{1}, \mu_{2}, \mu_{3}, \nu_{1}} g\left(\mu_{1}, \mu_{2}\right) g\left(v_{1}, \mu_{3}\right) \delta\left(\sigma_{2}^{1}, v_{1}^{1}\right) e\left(\sigma_{1}^{1}, \sigma_{3}^{1}\right)_{[1]} \cdots \delta\left(\sigma_{2}^{M}, v_{1}^{M}\right) e\left(\sigma_{1}^{M}, \sigma_{3}^{M}\right)_{[M]} \\
& =\sum_{\mu_{1}, \mu_{2}, \mu_{3}} g\left(\mu_{1}, \mu_{2}\right) g\left(\mu_{2}, \mu_{3}\right) e\left(\sigma_{1}^{1}, \sigma_{3}^{1}\right)_{[1]} \cdots e\left(\sigma_{1}^{M}, \sigma_{3}^{M}\right)_{[M]} .
\end{aligned}
$$

Continuing this process of multiplication by $A(M)$, we obtain

$$
A^{(N-1)}(M)=\sum_{\mu_{1}, \cdots, \mu_{N}} \prod_{j=1}^{(N-1)} g\left(\mu_{j}, \mu_{(j+1)}\right) e\left(\sigma_{1}^{1}, \sigma_{N}^{1}\right)_{[1]} \cdots e\left(\sigma_{1}^{M}, \sigma_{N}^{M}\right)_{[M]} .
$$

We multiply this expression for $A^{(N-1)}(M)$ by the matrix $A(M)$, using the sets of binary variables $v_{1}$ and $v_{2}$

$$
A(M)=\sum_{v_{2}} \sum_{v_{1}} g\left(v_{2}, v_{1}\right) e\left(v_{2}^{1}, v_{1}^{1}\right)_{[1]} e\left(v_{2}^{2}, v_{1}^{2}\right)_{[2]} \cdots e\left(v_{2}^{M}, v_{1}^{M}\right)_{[M]} .
$$

As a result, we obtain the following expression for $A^{N}(M)$

$$
A^{N}(M)=\sum_{\mu_{1}, \cdots, \mu_{N}, v_{1}} \prod_{j=1}^{N-1} g\left(\mu_{j}, \mu_{(j+1)}\right) e_{[1]} g\left(\mu_{N}, v_{1}\right) e\left(\sigma_{1}^{1}, v_{1}\right)_{[1]} \cdots e\left(\sigma_{1}^{M}, v_{1}^{M}\right)_{[M]} .
$$

Calculating the trace of the right side of this equation by the Formula (10), we obtain the partition function (1) in the form (4).

Now we compute the transfer matrix $A(M)$ (14). The sum over all values of the set $\mu_{1}$ of the transfer matrix (14) can be written in the form $A(M)=Q+W$, where

$$
\begin{gathered}
Q=\exp (H) \sum_{\mu_{2}} e\left(1, \sigma_{2}^{1}\right)_{[1]} \exp \left(K_{1} \sigma_{2}^{1}\right) \prod_{i=1}^{M-1} e\left(\sigma_{2}^{i}, \sigma_{2}^{i+1}\right)_{[i+1]} \exp \left(K_{i+1} \sigma_{2}^{i+1}\right), \\
W=\exp (-H) \sum_{\mu_{2}} e\left(1, \sigma_{2}^{1}\right)_{[1]} \exp \left(-K_{1} \sigma_{2}^{1}\right) \prod_{i=1}^{M-1} e\left(\sigma_{2}^{i}, \sigma_{2}^{i+1}\right)_{[i+1]} \exp \left(-K_{1+1} \sigma_{2}^{i+1}\right) .
\end{gathered}
$$

The expression for $Q$ can be expressed through the product of matrices of size $2 \times 2$ with elements from indexed matrices. Let us write it in more detail

$$
\begin{aligned}
Q= & \exp (H) \sum_{\sigma_{2}^{1}} e\left(1, \sigma_{2}^{1}\right)_{[1]} \exp \left(K_{1} \sigma_{2}^{1}\right) \sum_{\sigma_{2}^{2}} e\left(1, \sigma_{2}^{1}\right)_{[2]} \exp \left(K_{2} \sigma_{2}^{2}\right) \cdots \\
& \sum_{\sigma_{2}^{M-1}} e\left(\sigma_{2}^{M-2}, \sigma_{2}^{M-1}\right)_{[M-1]} \exp \left(K_{M-1} \sigma_{2}^{M-1}\right) \sum_{\sigma_{2}^{M}} e\left(\sigma_{2}^{M-1}, \sigma_{2}^{M}\right)_{[M]} \exp \left(K_{M} \sigma_{2}^{M}\right),
\end{aligned}
$$

We now introduce the following measurands

$$
S^{i}=\left\{\left(e_{[i]} \exp \left(K_{i}\right), f_{[i]} \exp \left(-K_{i}\right)\right),\left(g_{[i]} \exp \left(K_{i}\right), h_{[i]} \exp \left(-K_{i}\right)\right)\right\},
$$

and denote by the symbols $u$ and $v$ the vectors $u=[1,0], v=[0,1]$. Then $Q$ takes the form

$$
Q=\exp (H) u \prod_{i=1}^{M} S^{i}\left(u^{\mathrm{T}}+v^{\mathrm{T}}\right) .
$$

We transpose this polynomial from indexed matrices by transposing the indexed matrices without changing their previous places in the polynomial, which is possible due to their commutation (6). Then we obtain the following expres- 
sion for $Q^{\mathrm{T}}$

$$
Q^{T}=\exp (H) u \prod_{i=1}^{M} r_{i}\left(K_{i}\right)\left\{\left(e_{[i]}, g_{[i]}\right),\left(f_{[i]}, h_{[i]}\right)\right\}\left(u^{\mathrm{T}}+v^{\mathrm{T}}\right),
$$

where the symbol $r\left(K_{i}\right)$ denotes a matrix that has the form

$$
r\left(K_{i}\right)=\left\{\left(\exp \left(K_{i}\right), 0\right),\left(0, \exp \left(-K_{i}\right)\right)\right\} .
$$

Using the commutation relations (13), we write the expression for $Q^{\mathrm{T}}$ in the form

$$
\begin{aligned}
Q^{\mathrm{T}} & =\exp (H) \prod_{i=1}^{M} r_{[i]}\left(K_{i}\right) \prod_{i=1}^{M-1} P_{[i+1,1]} u\left\{\left(e_{[1]}, g_{[1]}\right),\left(f_{[1]}, h_{[1]}\right)\right\}\left(u^{\mathrm{T}}+v^{\mathrm{T}}\right) \\
& =\exp (H) \prod_{i=1}^{M} r_{[i]}\left(K_{i}\right) \prod_{i=1}^{M-1} P_{[i+1,1]}\left(e_{[1]}+g_{[1]}\right)
\end{aligned}
$$

We introduce new notation

$$
\begin{gathered}
t\left(K_{i}\right)=\left\{\left(\exp \left(-K_{i}\right), 0\right),\left(0, \exp \left(K_{i}\right)\right)\right\}, \\
c(H)=\{(\exp (H), 0),(0, \exp (-H))\}, \\
b_{[i, 1]}\left(K_{i}\right)=e_{[1]} r_{[i]}\left(K_{i}\right)+h_{[1]} t_{[i]}\left(K_{i}\right)
\end{gathered}
$$

and, using the commutation relations and Formulas (6), (7), (11), we get

$$
Q^{\mathrm{T}}=P_{M}^{-1} r_{[1]}\left(K_{M}\right)\left(e_{[1]}+g_{[1]}\right) c_{[1]}(H) \prod_{i=1}^{M-1} b_{[i+1,1]}\left(K_{i}\right) .
$$

In the same way, we obtain the following expression for $W^{\mathrm{T}}$

$$
W^{\mathrm{T}}=P_{M}^{-1} t_{[1]}\left(K_{M}\right)\left(f_{[1]}+h_{[1]}\right) c_{[1]}(H) \prod_{i=1}^{M-1} b_{[i+1,1]}\left(K_{i}\right) .
$$

Then for $A^{\mathrm{T}}(M)=Q^{\mathrm{T}}+W^{\mathrm{T}}$ we obtain the formula

$$
A^{\mathrm{T}}(M)=P_{M}^{-1} a_{[1]}\left(K_{M}\right) c_{[1]}(H) \prod_{i=1}^{M-1} b_{[i+1,1]}\left(K_{i}\right),
$$

where the matrix $a\left(K_{M}\right)$ has the form

$$
a\left(K_{M}\right)=\left\{\left(\exp \left(K_{M}\right), \exp \left(-K_{M}\right)\right),\left(\exp \left(-K_{M}\right), \exp \left(K_{M}\right)\right)\right\}
$$

Hence we obtain the required expression for the transfer matrix $A(M)$ of the one-dimensional Ising model (1)

$$
A(M)=\prod_{i=1}^{M-1} b_{[i+1,1]}\left(K_{i}\right) c_{[1]}(H) a_{[1]}\left(K_{M}\right) P_{M} .
$$

\section{Some Questions of Numerical Model Analysis}

The resulting transfer matrix has a structure that makes it possible to use the well-known method of power iterations of the matrix very effectively to calculate its PEV. The effectiveness of this method is due to the extreme thinness of the transfer matrix and the existence of the PEV. For any $M$, the matrix of the permutation operator of indices has only one nonzero element equal to one in each 
row and each column, and the matrix that faces the matrix of the permutation operator of the indices is block-diagonal with blocks of size $2 \times 2$. On the other hand, the M-th power of the transfer matrix is the matrix with positive elements, and therefore Perron-Frobenius theorem [5] is fulfilled for it, according to which its PEV exists and depends analytically on the parameters of the matrix $A(M)$. The existence of the PEV is critical for the application of the method, and the sparseness of the transfer matrix makes it possible to construct an economical, fast-acting algorithm for calculating the PEV [6].

This kind of matrix was used to calculate the macro characteristics of two-dimensional Ising-type models with the interaction between nearest neighbors [4] [6]. There they were called the root transfer matrices (RTM) and the free energy $F^{(2)}$ of these two-dimensional models with RTM $A^{2}(M)$ was determined by the formula

$$
F^{(2)}=\lim _{M \rightarrow \infty} \lambda\left(A^{(2)}(M)\right),
$$

where $\lambda\left(A^{(2)}(M)\right)$ is the RTM of the matrix $A^{(2)}(M)$.

There are at least three cases in which the transfer matrix (15) in form exactly coincides with the RTM of the corresponding multidimensional models with the interaction between the nearest neighbors. The difference is that in one-dimensional models the parameter $M$ determines, in a certain sense, the distance between the interacting spins, and the parameter $M$ in multidimensional models is equal to the number of nodes of the two-dimensional lattice along the horizontal, while the number of nodes along the vertical is infinite. Let us consider these cases.

1) We choose the following values for the parameters $K_{i}$ in the transfer matrix (15): $K_{2}=\cdots=K_{M-1}=0, K_{1}=K, K_{M}=L$. In this case, the transfer matrix $A(M)$ of this model takes the form

$$
A(M)=b_{[2,1]}(K) c_{[1]}(H) a_{[1]}(L) P_{M} .
$$

This transfer matrix coincides, up to the notation, with the RTM of the two-dimensional Ising model on a square lattice with the interaction between nearest neighbors ([4], p. 128).

2) Let the parameters $K_{i}$ take the following values $K_{2}=\cdots=0, K_{M-1}=J$, $K_{1}=K, K_{M}=L$. Then the transfer matrix $B(M)$ of this model takes the form

$$
B(M)=b_{[M, 1]}(J) b_{[2,1]}(K) c_{[1]}(H) a_{[1]}(L) P_{M}
$$

and it, to within a similarity transformation, coincides with the RTM of the two-dimensional Ising model on a triangular lattice [6].

3) For the parameters $K_{i}$, which take the values $K_{2}=\cdots=0, K_{R-1}=0$, $K_{R}=J, K_{R+1}=0, K_{M-1}=0, K_{1}=K, K_{M}=L$, the transfer matrix $C(M)$ of the one-dimensional model takes the form

$$
C(M)=b_{[R+1,1]}(U) b_{[2,1]}(K) c_{[1]}(H) a_{[1]}(L) P_{M} .
$$


This transfer matrix for $M=\mathrm{RN}$ coincides with the RTM of the three-dimensional Ising model on a cubic lattice with the interaction between nearest neighbors ([4], p. 147).

Neither of these models exists for an exact solution with $H$ not equal to zero. In such cases, the necessary information on the behavior of macro-characteristics of the model can give a numerical analysis. And the links between one-dimensional and multidimensional models can be used to solve problems in studies of various models [7] [8]. In the iterative method, the rate of convergence to the limit is significantly influenced by the value of the PEV ratio to another higher absolute value of its own value. Calculations showed [6] that this ratio reaches its minimum value near points that in the limit as $M$ tends to infinity they tend to singular points of the model. Thus, the study of one-dimensional models can give an idea of a pattern of the arrangement of singular lines in the corresponding multidimensional models. It should be noted that the study of one-dimensional models is interesting also for and since their free energy is determined by the PEV of a matrix of finite size, their thermodynamic properties can in principle be investigated by numerical methods with the required accuracy.

\section{References}

[1] Cambardella, P., Dallmeyer, A., Maiti, K., Malagoli, M., Eberhard, W., Karn, K. and Carbone, C. (2002) Ferromagnetism in One-Dimensional Monoatomic Metal Chains. Nature, 416, 301-304. https://doi.org/10.1038/416301a

[2] Andriushchenko, P.D. and Nefedev, K.V. (2013) Magnetic Phase Transition in the Lattice Ising Model. Advanced Material Research, 718, 166-171. https://doi.org/10.4028/www.scientific.net/AMR.718-720.166

[3] Baxter, R.J. (1982) Exactly Solved Models in Statistical Mechanics. Academic Press, New York and London.

[4] Dmitriev, A.A., Katrakhov, V.V. and Kharchenko, Yu.N. (2004) Root Transfer Matrices in Ising Models. Nauka, Moscow. [In Russian]

[5] Horn, R. and Johnson, C. (1986) Matrix Analysis. Cambridge University Press, London.

[6] Katrakhov, V.V. and Kharchenko, Yu.N. (2006) Two-Dimensional Four-Line Models of the Ising Model Type. Theoretical and Mathematical Physics, 149, 1545-1558. https://doi.org/10.1007/s11232-006-0137-y

[7] Arnalds, U.B., Cyico, J., Stopfel, Y., Kapaklis, V., Barenbold, O., Verschuren, M.A., Volff, U., Neu, V., Bergman, A. and Hjorvarsson, B. (2016) A New Look on the Two-Dimensional Ising Model: Thermal Artificial Spins. New Journal of Physics, 18, Article ID: 023008. https://doi.org/10.1088/1367-2630/18/2/023008

[8] Fan, Y. (2011) One-Dimensional Ising Model with k-Spin Interactions. European Journal of Physics, 32, 1643. https://doi.org/10.1088/0143-0807/32/6/018 